\title{
Realtime Monitoring \& Source Tracking Water Pollution in Najafgarh Drain Before \& During COVID-19 Outbreak with FOREMS
}

\author{
A Case Study by PTESPL
}

\author{
Mr. Rohit Mayur Patole \\ Business Head \\ PT Ecological Services Private Limited \\ Mumbai, India
}

\author{
Mr. Mohit Negi \\ Jr. Service Executive \\ PT Ecological Services Private Limited \\ Gurgaon, India
}

\begin{abstract}
The case study depicts the outcome of implementing a Realtime Continuous Water Monitoring System designed specially for surface water bodies called FOREMS. The device helped in analysing various pollution index while it was installed in Najafgarh drain in Haryana. An IOT device is used to remotely $\log$ the pollution indices online for applied data analytics. The conclusion depicts a theoretical analysis against pollution source identification.
\end{abstract}

Keywords-Floating; Online; River; Surface; Effluent; Monitoring ; System ; IOT device; Pollution; Tracking

\section{INTRODUCTION}

Over the past years, due to rapid industrialisation and advanced agricultural activities environmental deterioration is becoming a major concern. Water is one of the most important and basic natural resource which is being used in industries, agricultural activities as well as for the domestic and commercial purpose. These activities leads to discharge of wastewater, hence, accurate analysis of wastewater is crucial for maintaining the health and safety of the people. Water from all three sectors i.e. domestic, industrial and agriculture contribute into wastewater loads in the Najafgarh Drain. For this purpose the Gurgaon stretch, near sector 107 of the Najafgarh Drain was chosen to be analysed for its wastewater quality parameters. On the same stretch of the Drain, treated wastewater from several Wastewater Treatment plant is also disposed.

The present study deals with the comparison between the wastewater quality before and after the input of the treated Industrial effluent water in the Drain. These results can provide a basis for further studies and planning of facilities that can aid to solve the problem of deterioration of the natural resources and environment.

One of the major issues regarding rejuvenating of the Najafgarh drain is the identification of illegal discharge sources or disposal locations if any. Tracking either of defaulting source industrial or sewage effluent that contributed to gross pollution levels. Referring GMDA reports, at least 80 million liters of untreated sewage flows directly into Gurugram's storm water drains every day, polluting rivers, damaging underground water table, posing a major health hazard toll to the nearby population.
The magnitude of toxicity in flowing water varies every half hour which makes it challenging to trace pollution exceeding event by conventional manual grab sampling. And even after an exceeding pollution index is detected, the water flows through the stretch due to the time slippage in sample analysis and taking action. Hence before finding a solution it is important to analyse the nature and magnitude of flowing toxicity on real-time basis, which led the need to install the system capable of measuring pollutants with respect to acceptable standards and registering that data for analyzing the variance with respect to time.

\section{THE CHALLENGE}

1. Tracing the source of pollution.

2. Attaining repeatable and valid data/trend to analyze its variation with respect to different periods.

3. Tracking Instances where toxic sludge and solid waste is directly dumped and tracing water quality when such events occur.

4. Tracking pollution data on odd timings and at various position upstream and downstream

5. Lack of sufficient data makes it real challenge to analyze or trace the sources that cause the pollution and per- form the preventive actions to predict recurrences.

\section{APPROACHING THE CHALLENGE}

1. A continuous monitoring mechanism need to be established at nodal points between each respective drain legs from where the effluent from treatment units merge with the primary drain channel

2. This monitoring mechanism must represent the best of precise and repeatable measurement of pollution indicating parameters, un-interrupted power and data transmission modules, intelligent software based alert mechanism, ability to measure sample at valid position all in one integrable system

3. This monitoring device will be collecting and transferring the data continuously for further analysis. 


\section{PROJECT DEPLOYMENT}

Haryana State Pollution Control Board (HSPCB) invited PTESPL through a letter of request to study the continuous quality data of Najafgarh drain. Subject to which a site visit was conducted. The factors for not installing a generic station type monitoring mechanism were observed few of which are as follows.

a. The level of water varied across the 24 hours due to time specific effluent discharged by treatment plants, making it difficult to fix sensors on a structure fixed to ground.

b. The inability of using pump as a sample transfer medium due to large proportion of debris flowing with water, it was easy for pipe conduit to choke every hour. Also the difficulty in fixing a suction hose at the centre position of the flowing channel.

c. Acquiring land, developing shelter, extending power and internet provisions at location were additional minor challenges and involved special permissions from several Government agencies.

To overcome the stated aspects FOREMS platform was selected and following sensors were installed onboard OPM300 to measure Chemical Oxygen Demand and Total Suspended Solids (BOD was correlated from COD), OPM pH to measure $\mathrm{pH}$ and Temperature, OPM Optical DO for Dissolved oxygen.

The location was finalised after joint visit with HSPCB and Gurugram Metropolitan Development Association (GMDA) near MCD toll plaza Sector 107 Gurgaon location at N28030'58.8”, E-76058'17.9" before the effluent enters Delhi Jurisdiction. FOREMS was installed by anchoring at the coast by a temporary fixture. The software was pre-configured and the dashboard of Data logging ECOLYSER was made ready. A Sim card of a carrier with optimum speed was integrated for transferring data over cloud. Before deploying the routine tests of system were performed some of which include operational and liquid testing of Solar, Charge controller, Battery block, DatCom, Sensors, Analysers components etc.

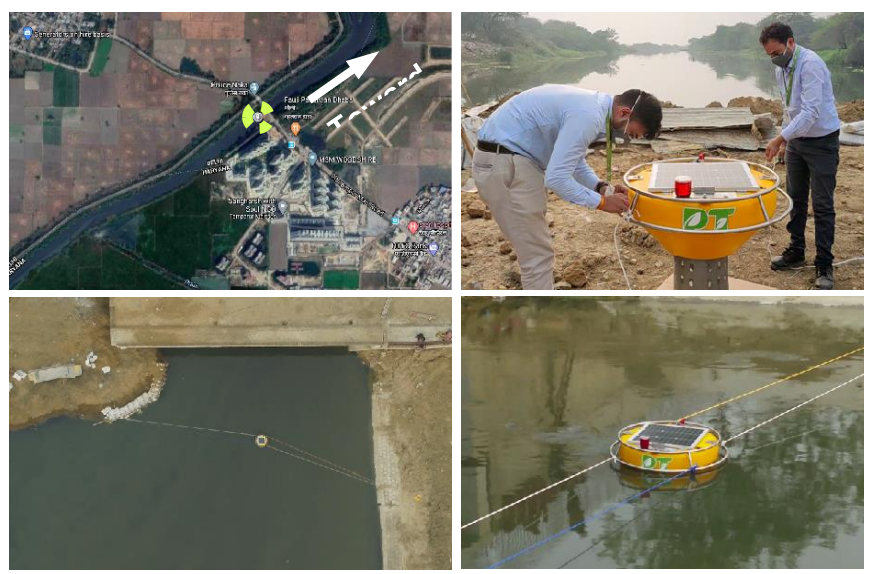

Figure 1. Description stated as clockwise starting from left top as location over satellite map, the team preparing to deploy FOREMS , and both images depicting it ready for operation
The in-situ deployed (floating over water) method of installation is adapted as per EPA guidelines for Continuous surface water quality monitoring system. Obtaining precise data would had been difficult incase a non-floating cabinet type type station was installed. For systems that use pumps for extracting samples it is difficult to qualify valid sample extraction which is at the centre of any flowing stream and from the flow column between $100 \mathrm{~mm}$ below the top surface and $200 \mathrm{~mm}$ above lower bed surface.

\section{V.OBSERVATIONS}

The team started collecting and analysing data from December 11th 2019 soon a_er the system was deployed. The system till April 11 has been running with $94 \%$ uptime. The installation month data helped in analyse about COD and TSS parameters exceeding in odd timings. The $\mathrm{pH}$ sensor was calibrated once every 2 weeks, OPM300 once every 2 months and OPM DO Once during the study tenure to fine tune the precision. The drain channel being a large flowing body that carries effluent from 6 different treatment plants, thus making it challenging to track the right defaulting source. The 6 treatment plants include 5 Sewage treatment plants of 150 and 120MLD plants at Behrampur , 50, 68, 100MLD at Dhanwapur and One Effluent Treatment plant of 55 MLD at Manesar. However due to COVID-19 outbreak, the entire nation was subject to lockdown since 23rd March 2020. This led to cutting off industrial effluent completely. The following data depicted in timeline of $21 \mathrm{st}$ March to $11^{\text {th }}$ April clearly indicates the drop in pollution parameters clearly concluding the sewage discharge is not the major pollution source.

\section{ANALYSIS OF DATA}

The data since January 11 to March 23 revealed a constant pattern. As per the pattern, the magnitudes of OPM300 sensor output for COD and TSS rise gradually by $30 \%$ of average value every day. The pattern starts rising by $8.00 \mathrm{pm}$ and flattens after highest peak, it settles down at the average base value by early morning $3.00 \mathrm{am}$. The data supposedly matches the flow meter readings installed at CETP and hence states a high probability of it being the main source of excessive pollution in Najafgarh.

And while this pattern continued, the TSS did not show any repeatable pattern. Around the clock other than this period the TSS values amplified by $15-20 \%$ (with respect to last 1 hour average data) every morning by 5.30 am for 1 hour. For the rest of the day the \% increase in COD and TSS followed a $20 \%$ identical average deviation rate, in other words, they both rose and drop at almost same rate, except while this specific pattern timing, thereby compelling the fact about a specific unique water matrix compared to average matrix throughout the day. The OPM $\mathrm{pH}$ values significantly crossed 7.5 mark by $5 \%$ during the occurrence of this pattern and throughout the rest of the day it varied in between 7.0 and 7.5. The OPM DO revealed the magnitudes fairly near 0 to 0.2 which can also be called out as dead value and coefficients resulting from error tolerance at $\pm 1 \%$ of Full scale of measurement. Dissolved oxygen values were found undeterminable but less than $1.0 \mathrm{mg} / \mathrm{l}$ when counter analysed through Laboratory sample analysis for 5 consecutive samples and hence match with the analyser data. 


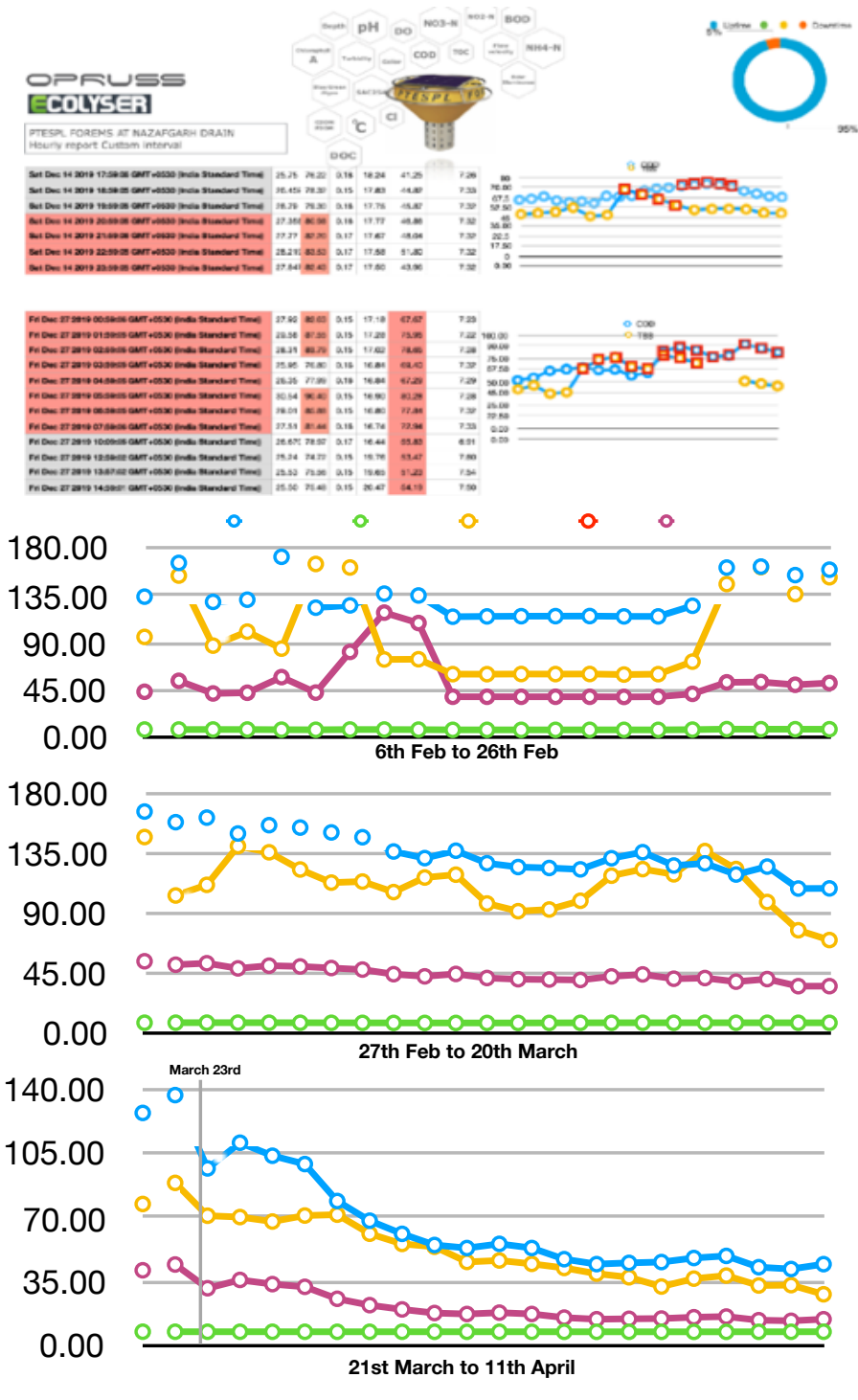

Figure 2. Data report extracted from ECOLYSER dashboard indicating intelligent pollution tracking capabilities

\section{CONCLUSION}

FOREMS deployed by PTESPL performed with $94 \%$ uptime and certainly without any continuous human assistance during critical period like the National Lockdown proving its reliability. Installation of FOREMS helped in tracking the pollution source of Najafgarh drain and that the sewage discharged from Gurgaon city contributed to only $35 \%$ of total water pollution. The regular pattern of pollution escalation taking place on odd timing as briefly stated in the observation, reveal a possible presence of either secret drains, or external dumping of toxic waste into the channel, this aspect may be analysed after deploying several more FOREMS at various nodes. Despite of severe weather conditions like unexpected rains causing high turbid flow in drain, near zero visibility for 10 continuous days of January, at this point the power module of FOREMS performed uninterruptedly by switching the system in low power mode. The notable part was to survive these natural conditions at the first occurrence.
Few Samples were manually grabbed on mid April 2020 in presence of GMDA authorities and were submitted in two different laboratories, one of it being NABL accredited and another internal lab of GMDA. Both the Lab results and FOREMS data were found to match by $97 \%$ accuracy. Due to lockdown event led by COVID-19, it was possible for us to observe data variance in two conditions of only Sewage effluent and Sewage and Industrial effluent.

The study concluded with a letter from Haryana State Pollution Control Board and also from Gurugram Metropolitan Development Authority, Haryana, acknowledging appreciation of the team's efforts for a successful and purposeful study.
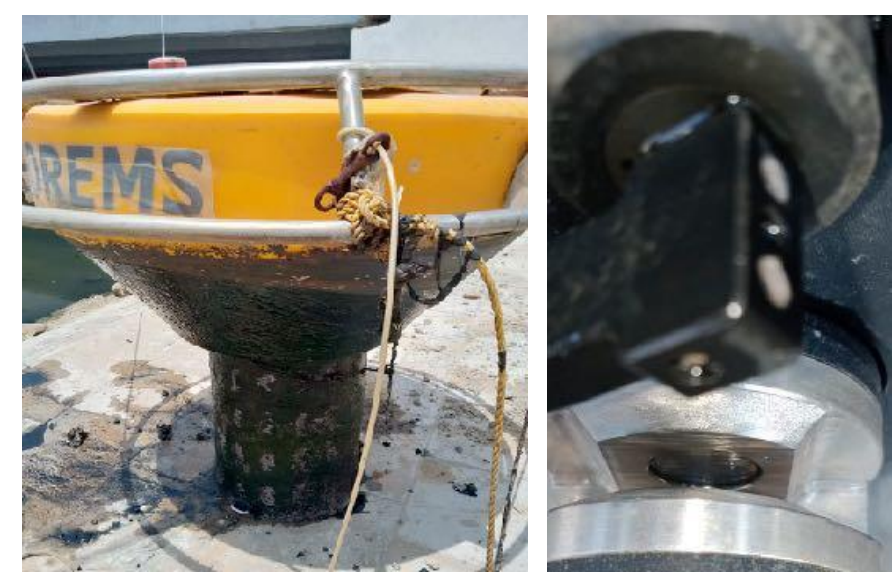

Figure 3. Image Depicting the importance of ACSC automatic cleaning mechanism

(Left) image of system retrieving after 5 months of deployment. (Right) OPM300 Sensor Cleaning efficiency of measuring aperture

The images indicate why only Conductive Surface cleaning systems work in such scenarios. In such studies it is important to choose an automatic cleaning mechanism technique, and it is better to not opt an external cleaning medium like compressed air or chemical solution whose performance could be interrupted by various environment factors. Also in such cases, pump based extractive sampling units are prone to fail due to excessive clogging and change in nature of effluent while passing through a conduit. It would have also been difficult to constantly stabilise the sampling inward point of conduit at the valid sampling column in such flow index.

\section{FUTURE SCOPE OF WORK}

- FOREMS can be used for the analysis of water quality based on the real time data between different stations further; it will help in identification of exact location of illegal discharge or polluting sources when placed at various location of upstream and downstream of junction points.

- Multiple sampling stations can chosen to study the 
decomposition rate of organic matter and its effect on DO of rivers or drains using FOREMS.

- Referring the EPA Water quality event detection system document, forecasting of pollution critical events by using a array of FOREMS is possible

- Additional parameters can be evaluated from same system due to flexible design. Parameters like ORP, TDS, CDOM, FDOM, NO3 if analyzed rightly we may be able to track the matrix of maximum pollution, can be called as source of pollution.

\section{ACKNOWLDGEMENT}

This document was developed by the PT Ecological Services Private Limited, with additional support provided by OPRUSS INDIA and ECOLYSER Team. A special thanks to the Haryana State Pollution Control Board \& Gurugram Metropolitan Development Authority for this opportunity The following individuals contributed to the development of this study

\section{PROJECT TEAM}

Pratik Thakur

Managing Director, PTESPL INDIA

Jay Shah

Director, PT Terado Engg

Rahul Manure

B.E Instrumentation, Production head

OPRUSS INDIA

Rohit Gupta

B.E Electronics, Asst. Service Head

PTESPL Mumbai

Sahil Babar

B.E Electrical, Production Engineer

OPRUSS INDIA

Mohit Negi

B.E Mechanical, Jr. Service Executive

PTESPL Gurgaon

Jayesh Singh

B.E Computer Engg, Sr Diagnostics Engineer

PT Terado Engg

Divya Jain

B.Sc IT, Support Engineer

PT Terado Engg

Sanket Sawant

UI/UX Designer, PT Terado Engg

Rohit Patole

Business head, PTESPL INDIA

\section{REFERENCES:-}

[1] In Situ Water Quality Monitoring ,USEPA, SESDPROC-111-R4 March 14, 2018

[2] Guidance for Building Online Water Quality Monitoring Stations For Source Water and Distribution System Moni- toring ,US EPA 817-B18-002 May 2018

[3] Online Source Water Quality Monitoring For Water Quality Surveillance and Response Systems ,US EPA 817-B-16- 003 September 2016

[4] Water Quality Event detection system for drinking water contamination warning system, US EPA/600/R-010/036, May 2010

[5] Guidelines for Water Quality Monitoring CPCB MINARS/27/200708

[6] Surface water sampling methods USEPA, SESDPROC-201-R3 Feb 19,2013

[7] green-ensys.org > publications WASTEWATER MANAGEMENT IN THE Najafgarh DRAIN BASIN

[8] Najafgarh drain-Sampling and analysis - India Environment Portal | News www.indiaenvironmentportal.org.in >

[9] Rejuvenating the Najafgarh basin - Smart Cities India expo www.smartcitiesindia.com >...

[10] Najafgarh drain-Sampling and analysis - GreenField Advanced Research

[11] Rejuvenation of Najafgarh waterway - Delhi Urban Art Commission 